\title{
Dynamic Interaction of Rotating Momentum Wheels with Spacecraft Elements
}

\author{
S. Shankar Narayan* P. S. Nair ${ }^{\dagger}$ and Ashitava Ghosal ${ }^{\ddagger}$
}

\begin{abstract}
In modern spacecraft with the requirement of increased accuracy of payloads, the on-orbit structural dynamic behaviour of spacecraft is increasingly influencing the design and performance of spacecraft. During the integrated spacecraft testing of one of the satellites, a strong coupling between rotating momentum wheels and an earth sensor was detected. This resulted in corruption of the earth sensor data at certain wheel speeds. This paper deals with the dynamic coupling problem of a rotating momentum wheel with its support brackets affecting other subsystems of spacecraft. As part of this investigation, extensive modal tests and vibration tests were carried out on the momentum wheel bracket assembly with wheels in stationary and rotating condition. It was found that effects of gyroscopic forces arising out of rotating wheels are significant and this aspect needs to be taken into account while designing the mounting brackets. Results of analysis and tests were used to redesign the bracket leading to significant reduction in the interaction and associated problems. A procedure for design of support structure using a low-order mathematical model is also shown.
\end{abstract}

\section{Introduction}

The effect of on-orbit structural dynamics on the performance of sensitive payloads are becoming increasingly important in the design of large, modern, complex, spacecraft. The source of vibratory disturbance on a spacecraft and its effects are well documented (see, for example, [1, 2, 3, 4, 5]). Vibratory disturbances can arise from rotating elements such as momentum wheels, reaction wheels, gyros, and solar array drives. In addition, elements like antenna-pointing mechanisms, and cryocoolers can also cause disturbances. This paper concentrates on the disturbances arising out of rotating components, in particular, those arising from momentum wheels. A momentum wheel (MW) is used for spacecraft attitude control and consists of a heavy rotating disk or wheel [6]. Even though the momentum wheels are very accurately balanced statically and dynamically, the high speed of operation $(\approx 4500$ to $5400 \mathrm{RPM})$, causes dynamic disturbances to the spacecraft $[8,9,10,11,12]$.

\footnotetext{
${ }^{*}$ Corresponding author. ISRO Satellite Centre, Vimanapura Post, Air Port Road, Bangalore 560017 , India. Email: sshankar@isac.gov.in

${ }^{\dagger}$ ISRO Satellite Centre, Vimanapura Post, Air Port Road, Bangalore 560 017, India. Email: psnair@isac.gov.in

${ }^{\ddagger}$ Dept. of Mechanical Engineering, Indian Institute of Science, Bangalore $560 \quad 012$, India. Email:asitava@mecheng.iisc.ernet.in
} 
While the dynamic forces imparted to the spacecraft are insignificant from structural dynamic considerations, it is important from the point of view of disturbance to sensitive instruments. Examples are scanning earth sensors, very high-resolution radiometers, and high-resolution remote sensing camera $[7,12]$. Some natural modes of these components may fall in the operating range of the momentum wheel speeds. The interaction of the rotating wheel and the mounting bracket, greatly alters the nature of disturbances itself. The focus of this paper is on the dynamic coupling between the momentum wheel and the supporting structure (bracket) which has not been adequately studied in literature.

There are two common configurations of momentum and reaction wheels. In the first type, the rotating disk or the flywheel is floating [14]. The mounting interface of the floating flywheel momentum wheel is on the outer rim as shown in figure 1. The mounting area on the spacecraft in this case is large. In the second type, the flywheel is attached to the base of the wheel assembly. This has smaller mounting interface area as shown in figure 2. This paper deals with the popular type 2 configuration shown in figure 2 .

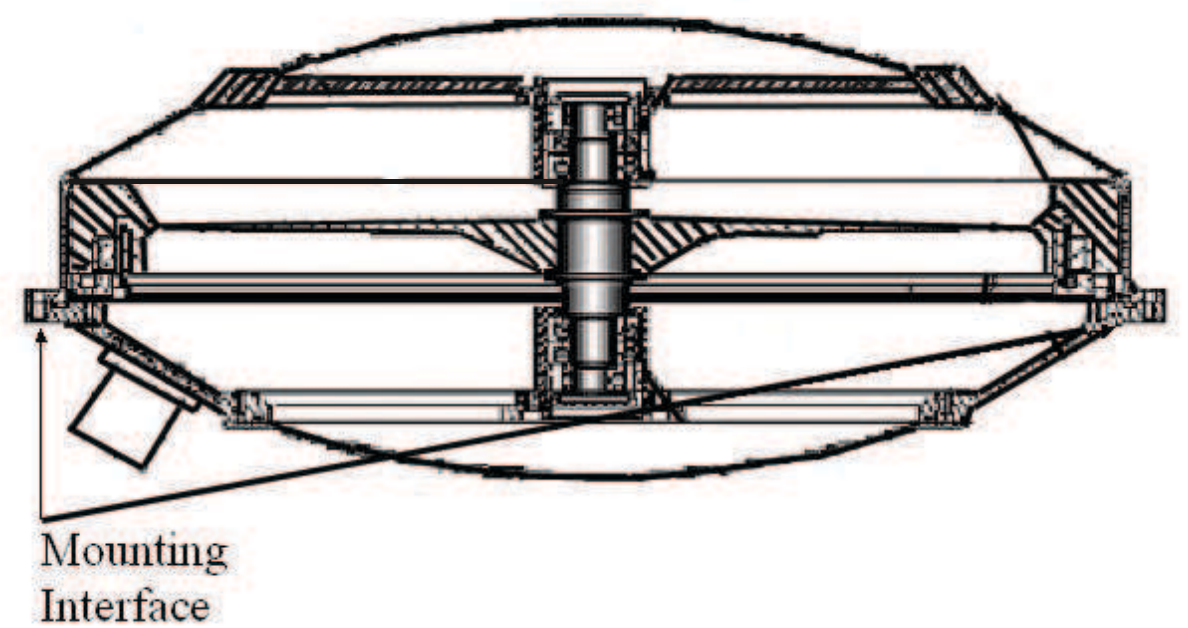

Figure 1: Wheel with large mounting interface diameter [14]

Extensive work has been reported on mathematical modeling of wheel disturbances. These models are either empirical or analytical $[11,12,13]$. Some of these models are derived from experimentally measured forces transmitted by momentum/reaction wheel at different rotational speeds. The forces and moments are measured by using a force plate. Davis et al., [8] have proposed steady state models of disturbances due to reaction wheels in the Hubble Space Telescope. 
Masterson $[14,15]$ has developed an empirical and analytical disturbance model for reaction wheel using measured data. These mathematical models are used in spacecraft jitter analysis. In the work by Masterson $[14,15]$, the configuration of momentum wheel of the type shown in figure 1 is used. The effect of coupling of the wheel rotation and the structural dynamics of wheel-housing bracket is not brought out. Elias $[18,19]$ has studied the interaction of momentum wheel with space structure, for the configuration shown in figure 2, using impedance techniques including the gyroscopic terms. However, the influence of gyroscopic terms on the structural dynamics of momentum wheel mounting bracket is not brought out in this case also.

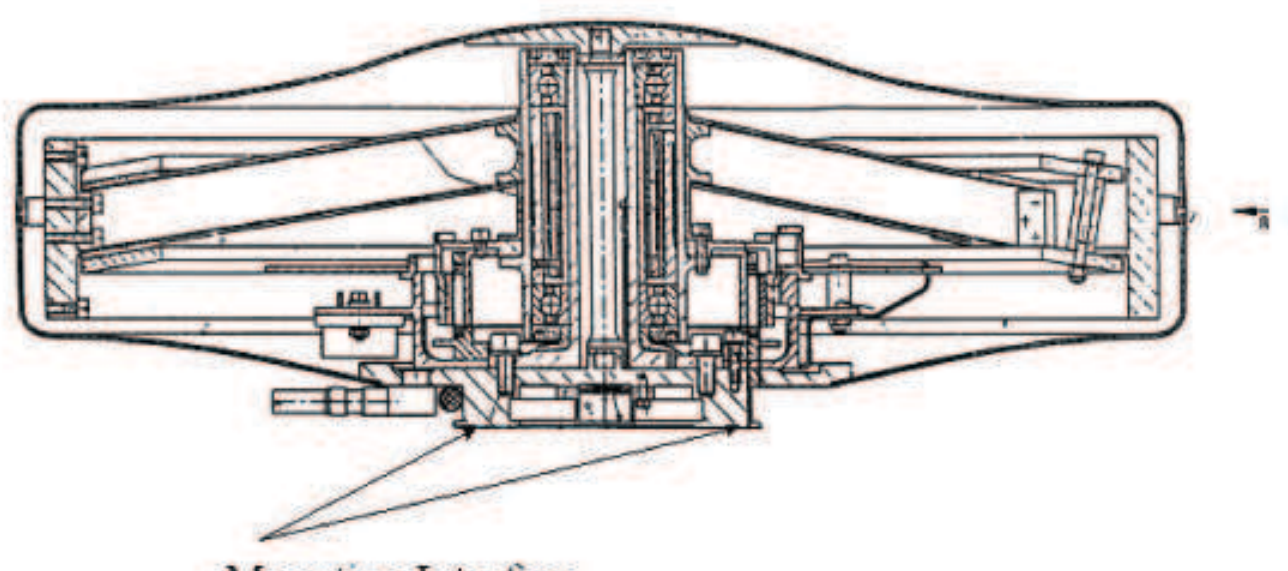

Mounting Interface

Figure 2: Wheel with smaller mounting interface diameter [16]

The present work is based on the extensive experimental and theoretical studies done on momentum wheel brackets of a geo-stationary spacecraft developed by Indian Space Research Organization (ISRO). During integrated spacecraft testing of the spacecraft, it was found that MW operations at certain speed ranges were significantly affecting the earth sensor ${ }^{1}$ output. Extensive tests were conducted to understand the nature of modes of spacecraft excited during the rotation of the momentum wheel. It was found that the frequencies measured with a stationary MW did not match the results with a rotating momentum wheel, implying a significant coupling between the rotating momentum wheel and the supporting brackets. Major contribution of this paper is the analysis of the coupling between the momentum wheel and the bracket. Design of the bracket, based on

\footnotetext{
${ }^{1}$ The earth sensor gives the deviation of spacecraft with respect to earth in terms of roll and pitch angular rates. It consists of a scanning mirror mounted on a flexure. The flexure has definite natural modes which are measured/estimated earlier.
} 
the analysis, which resulted in the disturbances being shifted outside the operating range of the momentum wheel, is also of interest to spacecraft designers.

This paper is organized into five sections. In section 2, the details of the configuration of the momentum wheel and the experimental data are presented. In section 3, details of the mathematical models and results of numerical analysis are given. The effect of changing different parameters of the bracket is also studied. The use of the results of analysis in section 3 for design modifications of the bracket are discussed in section 4. The experimental results on the modified bracket are discussed in section 4. Conclusions are given in section 5 .

\section{Momentum wheel configuration and experimental studies}

In a typical three-axis stabilized communication spacecraft, two-momentum wheels and one reaction wheel are used for attitude control [23]. The momentum wheels (MW) are attached to the spacecraft structure using brackets. In one of the geo-stationary spacecraft developed by ISRO, the wheel mounting bracket consisted of an aluminum honeycomb sandwich plate and a machined part, attached to the central thrust cylinder. A circumferential stiffener positioned near the MW bracket attachment area [16] further stiffens the thrust cylinder locally. The aluminum honeycomb sandwich plate and the machined bracket constituted the wheel mounting bracket (see figure 3 and figure 4 for details ).

\section{$2.1 \quad$ Tests and observations}

During integrated spacecraft tests, large errors were observed in the roll rate of the scanning earth sensor. These errors were seen only in specific speed ranges. To troubleshoot this problem, vibration responses were measured near the earth sensor. Figure 5 shows the acceleration response plot. Corresponding earth sensor rates are given in figure 6 for wheel speeds from 3000 RPM to $5400 \mathrm{RPM}$ with a speed variation at the rate of $6 \mathrm{RPM} / \mathrm{sec}$. The abscissa in the figure 6 represents the time in minutes and the ordinate represents the error measured by the earth sensor in degrees. This clearly indicates, as the rpm changes, the error detected by the earth sensor changes and is in tune with that of acceleration response of the momentum wheel bracket. This gives the relation between the rpm, momentum wheel bracket response and error detected by earth sensor. It is clear from the plot (figure 5) that the maximum response at the earth sensor location occurs at 4320 RPM $(72 \mathrm{~Hz})$. Waterfall plot of the acceleration response near earth sensor clearly showed peak response occurring at around $72 \mathrm{~Hz}$. As higher harmonics were not present, it was clear that the response was due the unbalance in the momentum wheels. Beating phenomenon was also observed, as the two wheels are operating nearly at equal speeds. Earth sensor data also showed the effects 


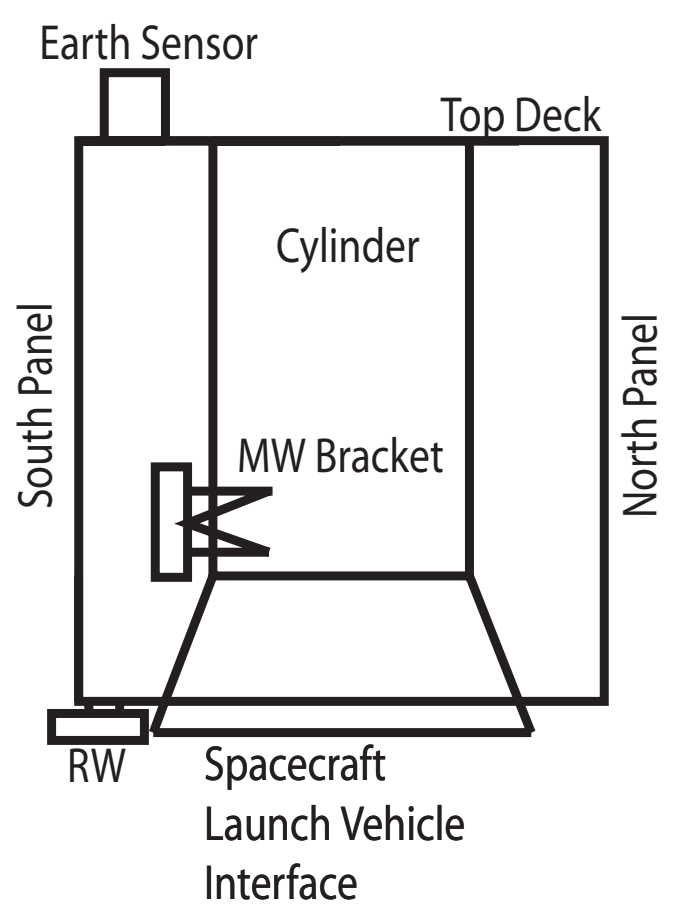

Figure 3: Schematic of Spacecraft with Earth Sensor and MW Bracket

on the roll error (see figure 6). These observations clearly indicated that the disturbances from the wheel caused the error in earth sensor output.

In order to study the problem corresponding to $72 \mathrm{~Hz}$ frequency, the responses at various points on the momentum wheel bracket were measured with only one wheel rotating (from $3000 \mathrm{RPM}$ to $5400 \mathrm{RPM}$ ). Responses obtained during these tests were similar to that obtained earlier for the two-wheel case. Experimental modal analysis was performed, with excitation at several locations on the bracket/momentum wheel. Figure 7 shows typical plot of frequency response function. It was very clear from these results, that the mode corresponding to $72 \mathrm{~Hz}$ was not excited in the modal tests. This was further confirmed by the experimental modal analysis. The $72 \mathrm{~Hz}$ mode appeared only when momentum wheel was rotating, and was traced to whirling of momentum wheel/bracket. The whirling mode of wheel and bracket was made clearly visible using a stroboscope synchronized with the momentum wheel rotation speed. Originally, the wheel-mounting bracket was designed so that its modes are away from the critical modes of the scanning earth sensor. However, the whirling effect altered the modes of the bracket and made it coincide with one of the modes of the scanning earth sensor, leading to large error in the output of earth sensor. 


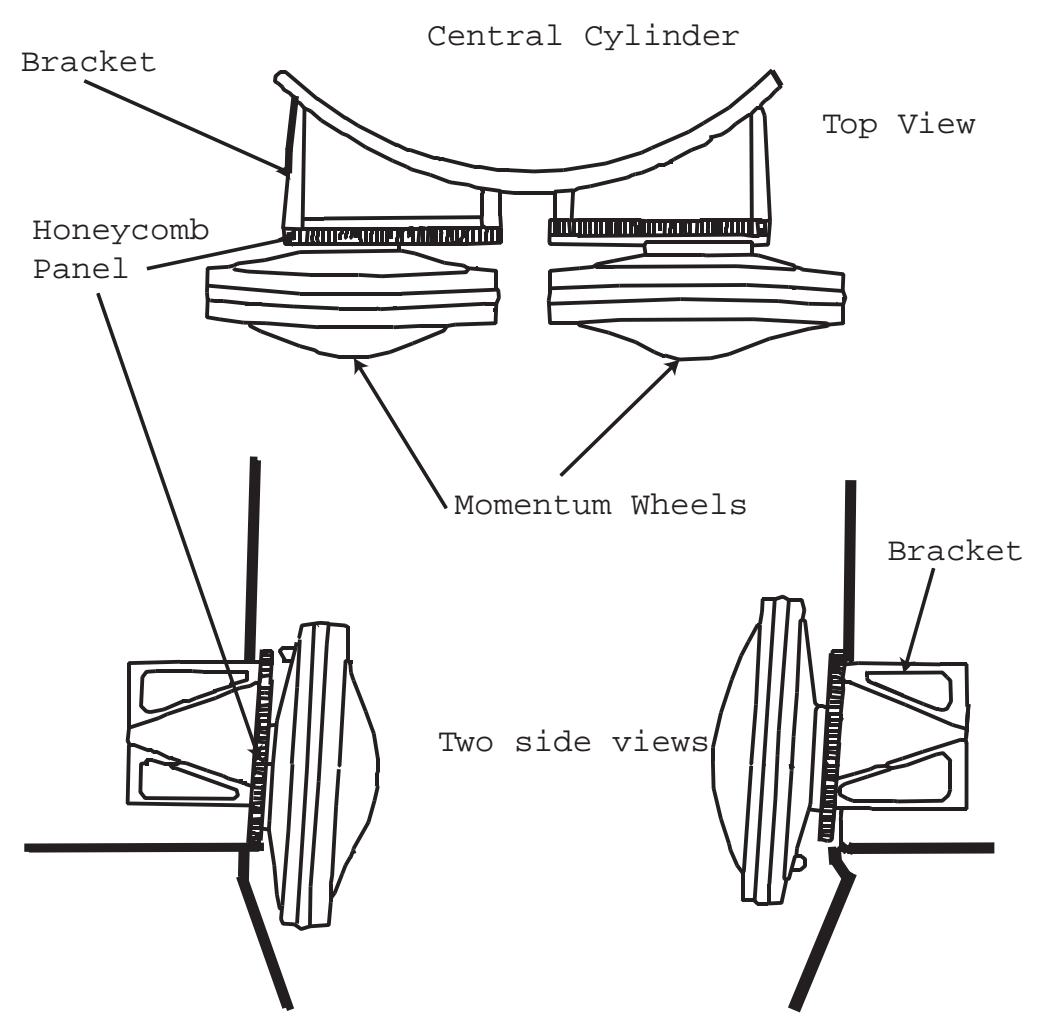

Figure 4: Momentum wheel configuration

\subsection{Results of experimental modal analysis}

Experimental modal analysis (EMA) was carried out to validate the finite element model shown in figure 8. For EMA, the MW bracket with MW is mounted on spacecraft. The bracket is excited using an electro-dynamic shaker. The frequency response functions are obtained by monitoring 12 locations along three orthogonal directions on MW bracket. The frequency response functions $(\mathrm{FRF})$ are then processed with the modal parameter estimation algorithm in I-DEAS@ Test software [21] to extract mode shapes. The first two mode shapes are shown on the right-hand side of figure 9 .

\section{Analytical studies}

To understand the effect of rotation of the momentum wheel on the structural dynamics, a finite element model of the bracket with wheel was generated using MSC NASTRAN(C). Figure 8 gives the finite element model of the bracket with the wheel. Wheel is modeled as lumped mass and inertia placed at its centre of gravity linked to the mounting points on the bracket by rigid links. A special 


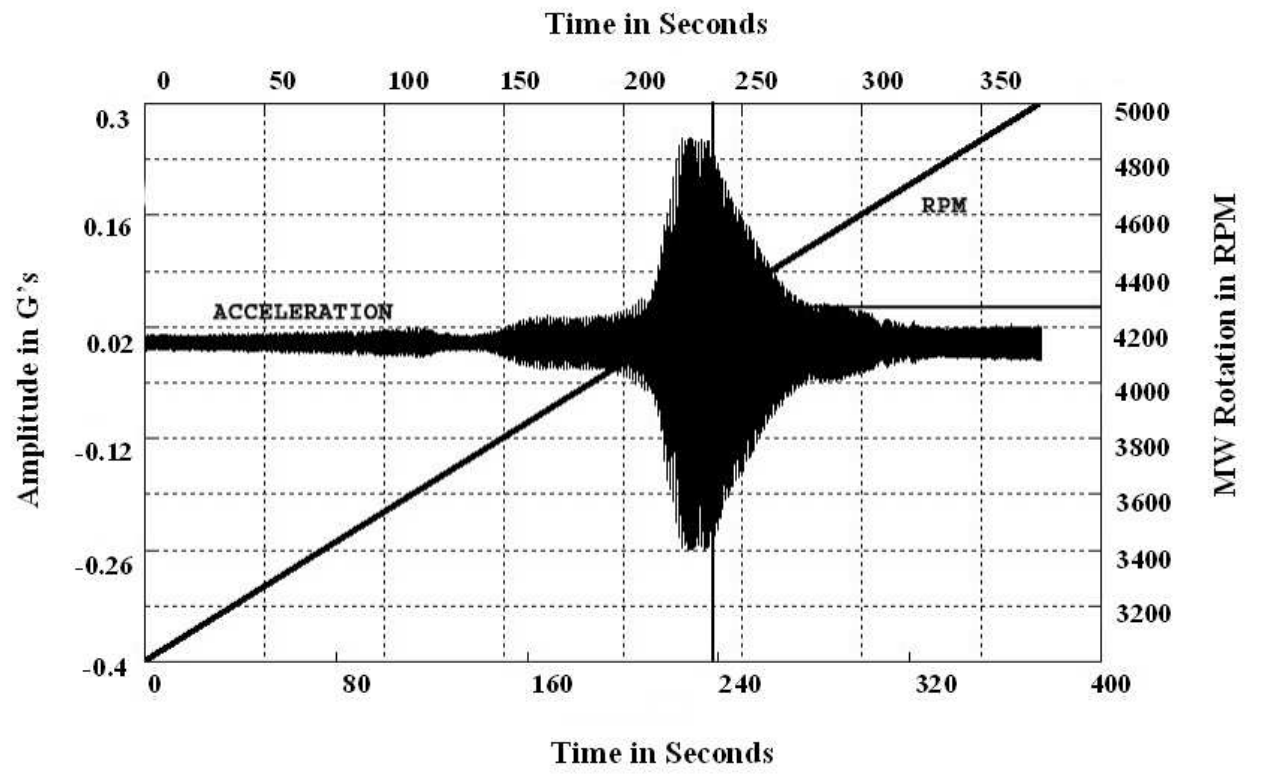

Figure 5: Acceleration response near earth sensor location

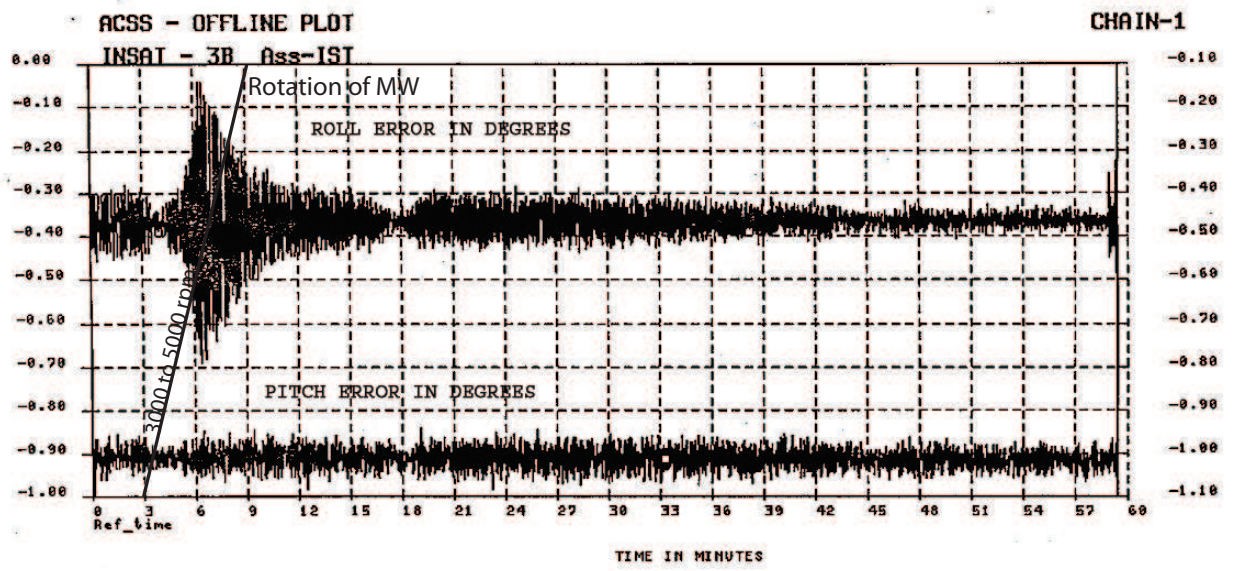

ES-1 Pitch Data ES-1 Roll Data

TIME IN MIMUTES

Figure 6: Error data (pitch and roll rates) from earth sensor

DMAP (Direct Matrix Abstraction Program) [20] sequence is added to the MSC NASTRAN(C) deck to include the effect of momentum wheel rotation. The mounting bracket along with the momentum wheel is modeled and is clamped at the six mounting interfaces points with spacecraft. The mounting interface flexibility of spacecraft main structure is not taken into account in this analysis. The finite element model contains 560 elements (QUAD4, lumped mass) and 4224 degrees 

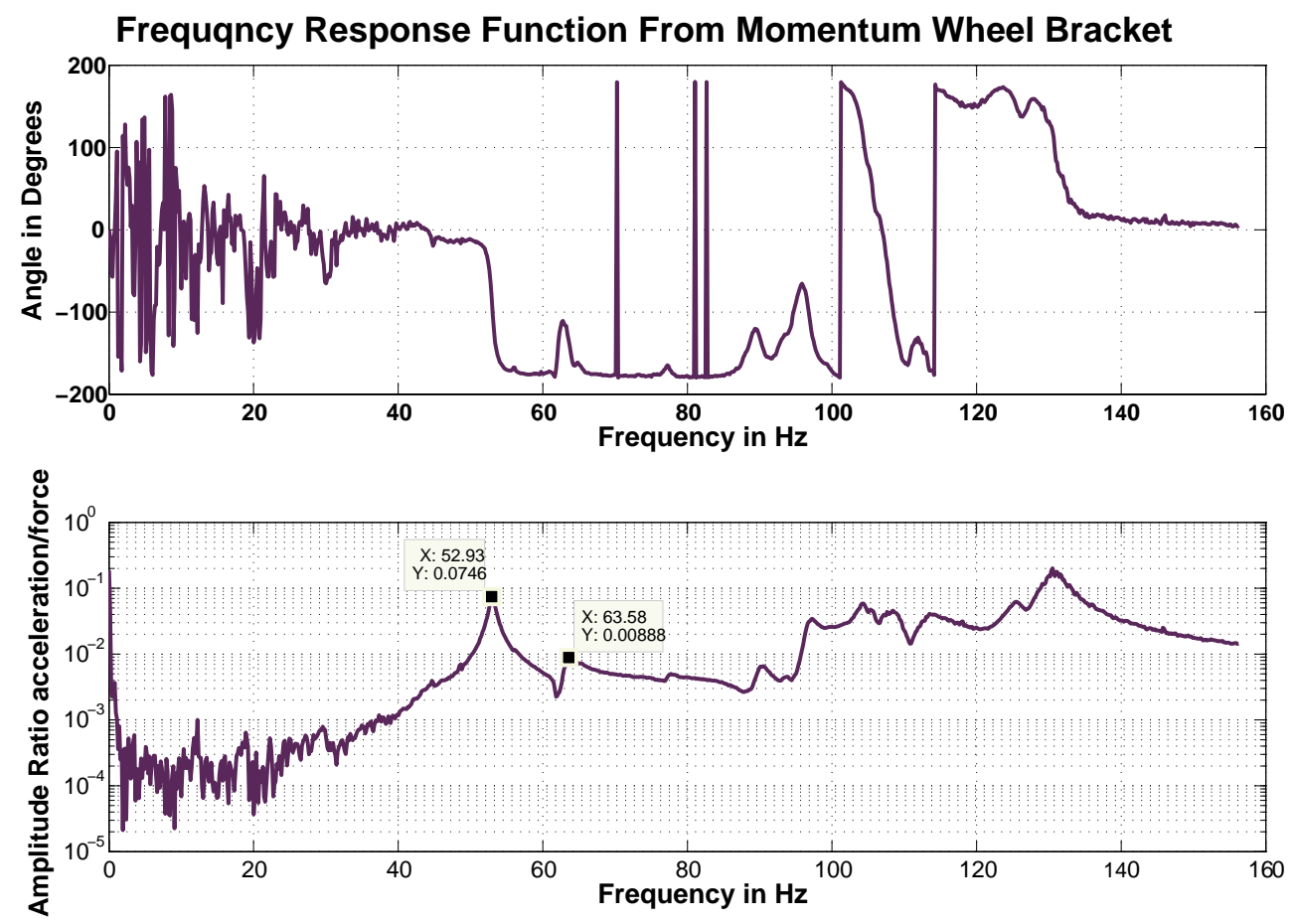

Figure 7: FRF from modal test measured on momentum wheel bracket

of freedom. The material properties for the face sheets, honeycomb core is taken from the material database. The wheel properties correspond to mass $8.1 \mathrm{~kg}, J_{x}=J_{y}=0.06972 \mathrm{kgm}^{2}$, and $J_{z}=$ $0.12763 \mathrm{kgm}^{2}$ [16]. The eigenvalue problem is solved first to obtain the natural frequencies without the effect of the of the momentum wheel. The left-hand side of figure 9 gives the first of two modes obtained from FEA, and, as observed by comparing the left- and right-sides of figure 9, there is a reasonable match between the FEA and the EMA results. Though neglecting interface flexibility may affect the modes involving the main structure, the basic structural dynamics arising out of local bracket deformations is captured in the FE model.

The mathematical model with wheel rotational effects is then studied. The complex eigenvalue analysis for this case was carried for different rotational speeds. Whirling of the MW with the bracket is clearly seen from the animated mode shapes. This confirmed that the gyroscopic forces generated by the rotating momentum wheel affects the structural dynamics of the bracket. The Campbell diagram for this case is plotted to bring out the change of natural frequency with respect to RPM. Figure 10 shows the Campbell diagram obtained from the complex eigenvalue analysis. The Campbell diagram shows that the first three modes of MW and bracket change with the rotation speed. A modal transient analysis (to obtain response at the same location as measured during 


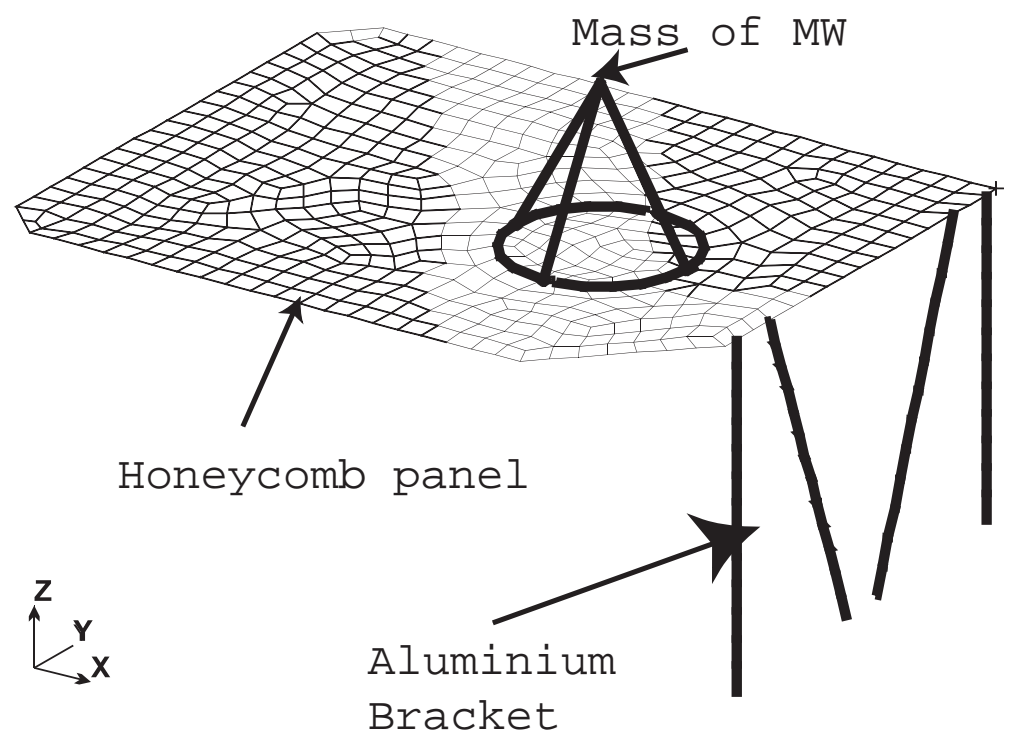

Figure 8: Finite element model of momentum wheel bracket

experiment) was carried out to identify the bracket mode excited due to the counter clockwise rotation of the wheel. The lowest mode gets excited due to rotation of the wheel and imparts significant interface forces on the spacecraft. The nature of the transient response was found to be very similar to that obtained from experiments on the spacecraft (see figure 5). A comparison of the frequencies obtained from FEM and EMA is given in Table 1. The difference in the frequencies for the first three modes between FEM and EMA is due to the difference in boundary conditions used FEM and EMA. During experimental modal anaysis, the MW bracket was fixed to the spacecraft. In the finite element analysis, the stiffness contribution from spacecraft at the interface of MW bracket with spacecraft is not incorporated. However, it is seen that the mode shapes match well as seen in figure 9 .

Table 1: Comparison of frequencies

\begin{tabular}{lcc}
\hline Node No. & Freq $(\mathrm{Hz})$ from FEM & Freq $(\mathrm{Hz})$ from EMA \\
\hline 1 & 67.69 & 52.94 \\
2 & 76.58 & 63.32 \\
3 & 130.4 & 80.12 \\
\hline
\end{tabular}

It is thus clear that the rotation of the momentum wheel affects the amplitude of disturbance indicated by response of the wheel and bracket. This in turn causes higher response at other 
spacecraft locations. At critical speeds, the response of the spacecraft is high. The low damping in the bracket also contributes to the higher response problem.

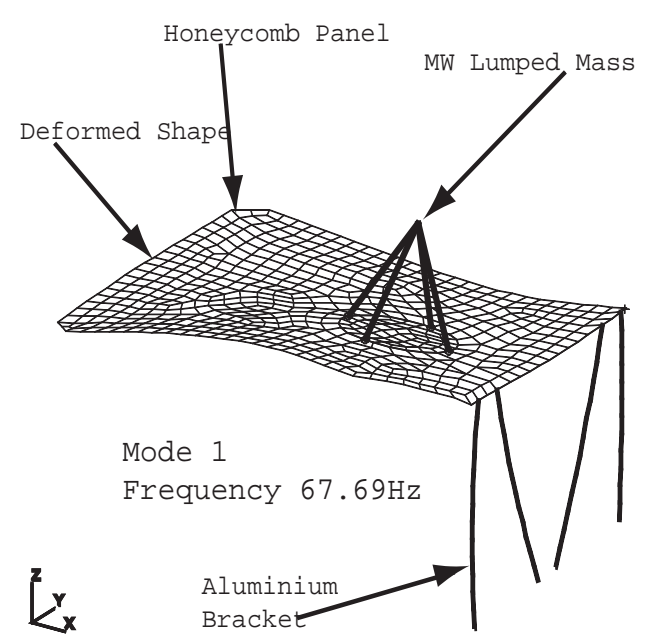

(a)

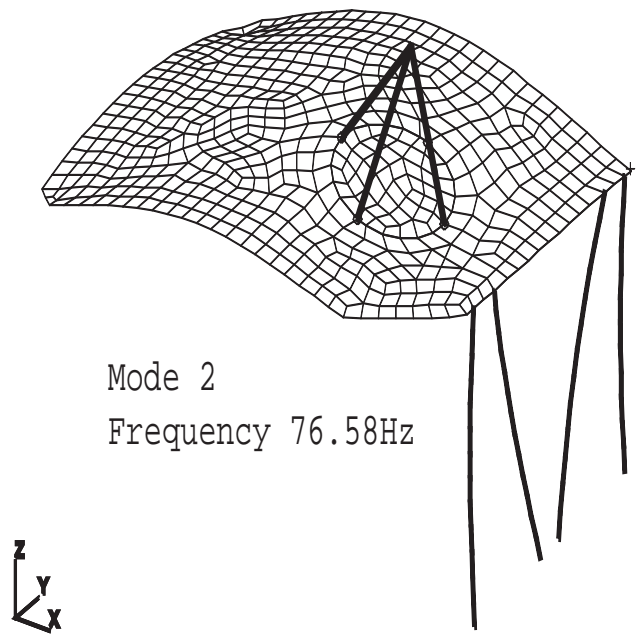

(c)

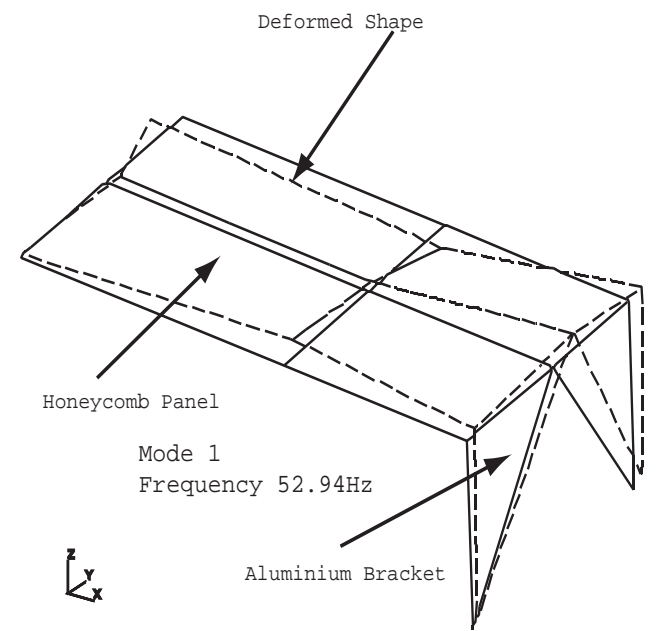

(b)

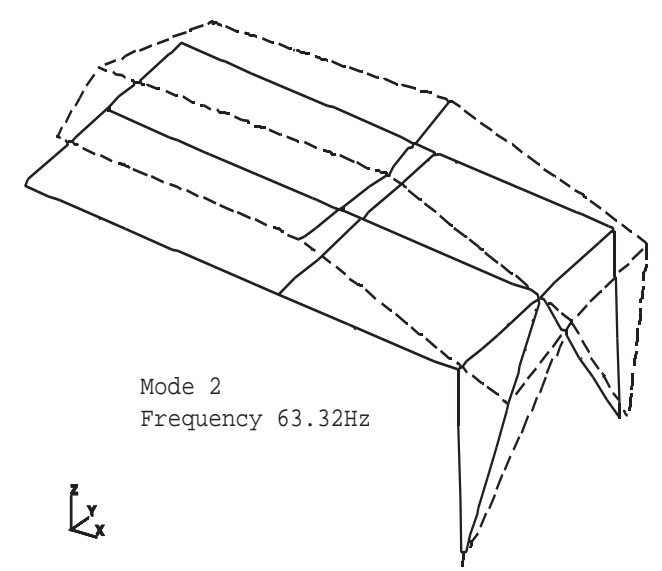

(d)

Figure 9: Mode shapes from FEM and EMA

\subsection{Lower-order mathematical model of bracket and momentum wheel}

As discussed earlier, momentum wheel - earth sensor interaction can make the attitude rates erroneous and the requirement is to minimize this interaction. Minimizing interaction can be done in many ways. One approach is to make the stiffness of the bracket very low so as to make it behave as an isolator. Another approach is to shift the whirling frequency of the bracket (during 
rotation of momentum wheel) well above the operating speed range of the wheel. The first option is structurally not feasible, as the wheel bracket has to withstand the high launch loads. The second option requires a redesign of the bracket and is easily feasible. The second option was chosen by us.

In order to redesign the existing wheel bracket, a lower-order model, which captures the essential design parameters and dynamic characteristics, is first derived. This is especially useful to avoid use of a large finite element model of the wheel-bracket for the iterative design process. This lower-order model is also useful for the spacecraft level studies.

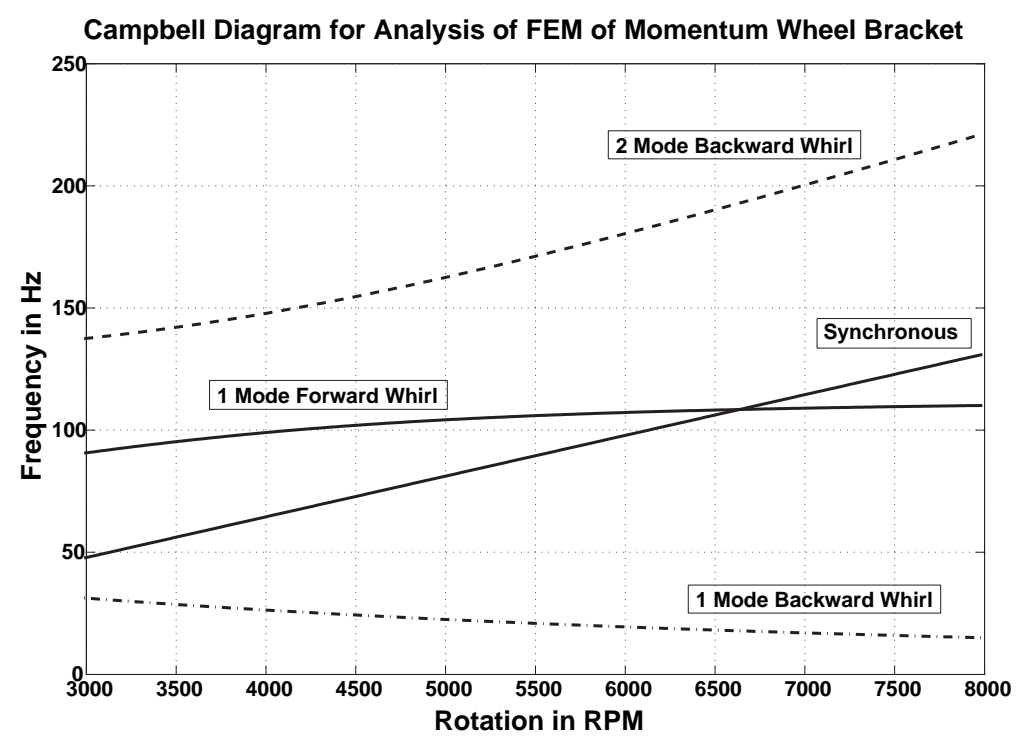

Figure 10: Campbell diagram from FEM analysis of momentum wheel bracket

The bracket consists of a honeycomb panel supported on two sides. It is connected to the central thrust cylinder - one side is directly connected and other side by a machined aluminum member. This bracket is modelled as a six-degree-of-freedom spring connecting wheel with spacecraft (shown in figure 3 and figure 4). For this study the MW can be considered as a rigid mass, with specified static and dynamic unbalances. The wheel is elastically supported at the 6 mounting interface points by linear springs. The mass of mounting bracket assembly is small compared to the wheel mass and hence is neglected. The three linear spring stiffness are denoted by $k_{x_{i}}, k_{y_{i}}, k_{z_{i}}, i=1 \cdots 6$, and the rotational stiffnesses about three mutually perpendicular axes are denoted by $k_{m x_{i}}, k_{m y_{i}}, k_{m z_{i}}, i=$ $1 \cdots 6$. These are shown schematically in figure 11 . The inertia properties are the mass, $m$, and mass momentum of inertia, $J_{x}, J_{y}, J_{z}$ with respect to the reference axes and at the centre of mass. The angular speed of the wheel is denoted by $\omega$ in radians/sec. The static unbalance is caused 
by the offset of centre of mass of the wheel from the axis of rotation and is modeled as mass, $m_{s}$, located at a radius, $r_{s}$. Misalignment of the principal axes of inertia of the wheel with respect to spin axis leads to dynamic unbalance. This is modeled as two equal masses, $m_{d}$, placed diametrically opposite at a radial distance, $r_{d}$, and axial distance, $h$, from the wheel mass centre.

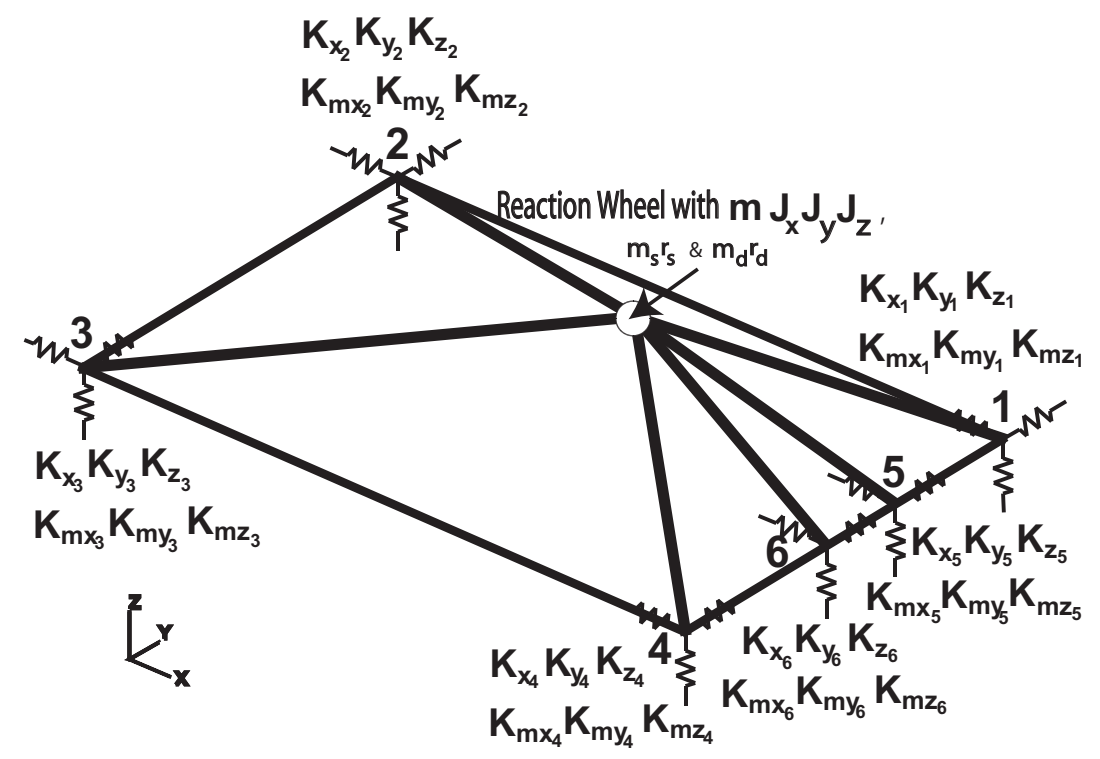

Figure 11: Momentum wheel mounting bracket modeled as springs

The equation of motion of the wheel-bracket combination is derived using Lagrange formulation and these can be written in a compact form as

$$
[\mathrm{M}]\{\ddot{\mathbf{x}}\}+[G]\{\dot{\mathbf{x}}\}+[K]\{\mathbf{x}\}=\mathbf{F}
$$

where the $6 \times 1$ vector $\mathbf{x}$ denote the generalized variables corresponding to the 6 degrees-of-freedom (dof) $\left(x, y, z, \theta_{x}, \theta_{y}, \theta_{z}\right)^{T}$, and the mass matrix and the gyroscopic matrix are as given below.

$$
[M]=\left[\begin{array}{cccccc}
m & 0 & 0 & 0 & 0 & 0 \\
0 & m & 0 & 0 & 0 & 0 \\
0 & 0 & m & 0 & 0 & 0 \\
0 & 0 & 0 & J_{x} & 0 & 0 \\
0 & 0 & 0 & 0 & J_{y} & 0 \\
0 & 0 & 0 & 0 & 0 & J_{z}
\end{array}\right]
$$




$$
[G]=\left[\begin{array}{cccccc}
0 & 0 & 0 & 0 & 0 & 0 \\
0 & 0 & 0 & 0 & 0 & 0 \\
0 & 0 & 0 & 0 & 0 & 0 \\
0 & 0 & 0 & 0 & -\omega J_{z} & 0 \\
0 & 0 & 0 & \omega J_{z} & 0 & 0 \\
0 & 0 & 0 & 0 & 0 & 0
\end{array}\right]
$$

The $6 \times 6$ stiffness matrix $[K]$ is given by

$$
\left[\begin{array}{llllll}
\sum k_{x_{i}} & 0 & 0 & 0 & \sum k_{z_{i}} z_{i} & -\sum k_{x_{i}} y_{i} \\
0 & \sum k_{y_{i}} & 0 & \sum k_{y_{i}} z_{i} & 0 & -\sum k_{y_{i}} x_{i} \\
0 & 0 & \sum k_{z_{i}} & \sum k_{z_{i}} y_{i} & -\sum k_{z_{i}} x_{i} & 0 \\
0 & -\sum k_{y_{i}} z_{i} & \sum k_{z_{i}} y_{i} & (*)_{1} & -\sum k_{z_{i}} x_{i} y_{i} & -\sum k_{y_{i}} x_{i} z_{i} \\
\sum k_{x_{i}} z_{i} & 0 & -\sum k_{z_{i}} x_{i} & -\sum k_{z_{i}} x_{i} y_{i} & (*)_{2} & -\sum k_{x_{i}} y_{i} z_{i} \\
-\sum k_{x_{i}} y_{i} & \sum k_{y_{i}} x_{i} & 0 & -\sum k_{y_{i}} x_{i} z_{i} & -\sum k_{x_{i}} y_{i} z_{i} & (*)_{3}
\end{array}\right]
$$

where the summation is over $i=1$ to 6 and the terms $(*)_{1},(*)_{2}$ and $(*)_{3}$ are given as $\sum\left(k_{y_{i}} z_{i}^{2}+\right.$ $\left.k_{z_{i}} y_{i}^{2}+k_{m x_{i}}\right), \sum\left(k_{z_{i}} x_{i}^{2}+k_{x_{i}} z_{i}^{2}+k_{m y_{i}}\right)$, and $\sum\left(k_{x_{i}} y_{i}^{2}+k_{y_{i}} x_{i}^{2}+k_{m z_{i}}\right)$, respectively.

The right-hand side forcing term $\mathbf{F}$ is given by

$$
\mathbf{F}=\left\{\begin{array}{c}
-m_{s} r_{s} \omega^{2} \sin (\omega t) \\
m_{s} r_{s} \omega^{2} \cos (\omega t) \\
0 \\
m_{d} r_{d} h \omega^{2} \cos (\omega t) \\
m_{d} r_{d} h \omega^{2} \sin (\omega t) \\
0
\end{array}\right\}
$$

where $m_{s} r_{s}$ and $m_{d} r_{d} h$ represent the static and dynamic unbalance respectively as explained earlier.

It is seen that the forcing terms on the right-hand side of the equations correspond to both the translations and rotations and are harmonic functions with a frequency equal to the wheel speed. It is also clear that the matrix $[G]$ changes with rotational speed.

\subsection{Numerical analysis}

In order to have a good initial estimate of the stiffness of each spring used in the lower-order model, component mode synthesis [22] is adopted. The basis for this reduction method is the transformation matrix known as Craig-Bampton coordinate transformation. This reduces the finite element stiffness matrix into an assembly of an interface stiffness matrix and diagonal modal stiffness matrix. The initial estimate for the stiffness for each spring here is based on the interface stiffness matrix. A brief review of the component mode synthesis following the approach by Craig and Bampton [22] and its application to arrive at the spring stiffnesses in the lower-order model of the bracket is given here. 
The equations of motion of a general dynamical system without damping is given by

$$
[M]\{\ddot{\mathbf{x}}\}+[K]\{\mathbf{x}\}=\mathbf{F}
$$

In the first step, the physical degrees of freedom are separated into a set of non-interface (interior) coordinates $x_{N}$ and a set of interface degrees of freedom coordinates $x_{I}$. The matrices and vectors in equation (6) are then partitioned as

$$
\left[\begin{array}{cc}
M_{N N} & M_{N I} \\
M_{N I}^{T} & M_{I I}
\end{array}\right]\left\{\begin{array}{c}
\ddot{\mathbf{x}}_{N} \\
\ddot{\mathbf{x}}_{I}
\end{array}\right\}+\left[\begin{array}{cc}
K_{N N} & K_{N I} \\
K_{N I}^{T} & K_{I I}
\end{array}\right]\left\{\begin{array}{c}
\mathbf{x}_{N} \\
\mathbf{x}_{I}
\end{array}\right\}=\left\{\begin{array}{c}
\mathbf{F}_{N} \\
\mathbf{F}_{I}
\end{array}\right\}
$$

where typically the non-interface forces $F_{N}$ are zero.

Let $\phi_{N N}$ denote the matrix of fixed-interface modes of the system normalized with respect to mass matrix. The modes are obtained using a standard eigenvalue solution process with interfaces fixed using $\phi_{C N}$ matrix denote the matrix of constraint modes, i.e., the rigid body displacement vector. The physical degrees of freedom $\mathrm{x}$ and modal degrees of freedom can be written as

$$
\mathbf{x} \equiv\left\{\begin{array}{c}
\mathbf{x}_{N} \\
\mathbf{x}_{I}
\end{array}\right\}=\left[\begin{array}{cc}
\phi_{N N} & \phi_{C N} \\
0 & I
\end{array}\right]\left\{\begin{array}{c}
\mathbf{q}_{N} \\
\mathbf{x}_{I}
\end{array}\right\}
$$

where $\mathbf{q}_{N}$ are the modal degrees of freedom and the matrix

$$
\left[\begin{array}{cc}
\phi_{N N} & \phi_{C N} \\
0 & I
\end{array}\right]
$$

is known as the Craig-Bampton transformation matrix.

Using this transformation matrix in equation (7), leads to

$$
\left[\begin{array}{cc}
I & \tilde{M}_{N I} \\
\tilde{M}_{N I}^{T} & \tilde{M}_{I I}
\end{array}\right]\left\{\begin{array}{c}
\ddot{\mathbf{q}}_{N} \\
\ddot{\mathbf{x}}_{I}
\end{array}\right\}+\left[\begin{array}{cc}
\Omega_{n}^{2} & 0 \\
0 & \tilde{K}_{I I}
\end{array}\right]\left\{\begin{array}{c}
\mathbf{q}_{N} \\
\mathbf{x}_{I}
\end{array}\right\}=\left\{\begin{array}{c}
\phi_{N N}^{T} \mathbf{F}_{N} \\
\phi_{C N}^{T} \mathbf{F}_{N}+\mathbf{F}_{I}
\end{array}\right\}
$$

where

$$
\begin{aligned}
\phi_{C N} & =-K_{N N}^{-1} K_{N I} \\
\tilde{K}_{I I} & =K_{N I}^{T} \phi_{C N}+K_{I I} \\
\tilde{M}_{I I} & =\phi_{C N}^{T}\left[M_{N N} \phi_{C N}+M_{N I}\right]+M_{N I} \phi_{C N}+M_{I I} \\
\tilde{M}_{N I} & =\phi_{N N}^{T}\left[M_{N N} \phi_{C N}+M_{N I}\right]
\end{aligned}
$$

with $\tilde{M}_{N I}$ as the coupling matrix, and $\tilde{K}_{I I}$ as the interface stiffness matrix.

MSC NASTRAN@ finite element analysis tool was used to perform the component mode synthesis. The large matrices obtained from the finite element analysis were reduced to matrices $\tilde{K}_{I I}, \tilde{M}_{I I}$ and $\tilde{M}_{N I}$. As there are six constraint points in the finite element model, the interface 
stiffness matrix $K_{I I}$ is a $36 \times 36$ fully populated matrix. All the elements of this matrix are not used for the initial estimate of stiffnesses used in the lower-order model. Coupled off-diagonal stiffness terms are neglected for simplicity. From the mode shapes of the finite element analysis and experimental modal analysis, one observes that the first two modes are anti-symmetric. The honeycomb panel is free to move in the lateral direction. It is clear, again from the FEM and EMA, that the mode shape is primarily controlled by $k_{z_{i}}, i=1 \cdots 6$ and hence, only the stiffnesses in the $Z$ direction are chosen for the initial estimate for the lower-order model. The stiffness values used from the interface stiffness matrix are given in Table 2. It may be noted that there is no unique way of deciding which stiffnesses to keep. However, as observed in the extensive simulations (discussed later), the choice of keeping only the $Z$ components of the stiffnesses is adequate for this study.

Table 2: Comparison of stiffness

\begin{tabular}{lcc}
\hline $\begin{array}{l}\text { Stiffness } \\
\text { Nomenclature }\end{array}$ & $\begin{array}{c}\text { Stiffness in } \mathrm{N} / \mathrm{m} \\
\text { from Component Mode } \\
\text { Synthesis }\end{array}$ & $\begin{array}{c}\text { Stiffness in N/m } \\
\text { after iterations }\end{array}$ \\
\hline$k_{z_{1}}$ & 496528 & 400000 \\
$k_{z_{2}}$ & 288109 & 200000 \\
$k_{z_{3}}$ & 280669 & 200000 \\
$k_{z_{4}}$ & 502330 & 400000 \\
$k_{z_{5}}$ & 622066 & 350000 \\
$k_{z_{6}}$ & 632273 & 350000 \\
\hline
\end{tabular}

Keeping only the $k_{z_{i}}$ 's in equation (1), it is observed that the set of six equations in the lowerorder model further reduce to only three equations. These are given as

$$
\begin{aligned}
& {\left[\begin{array}{ccc}
m & 0 & 0 \\
0 & J_{x} & 0 \\
0 & 0 & J_{y}
\end{array}\right]\left\{\begin{array}{c}
\ddot{z}_{\ddot{\theta}_{x}} \\
\ddot{\theta}_{y}
\end{array}\right\}+\left[\begin{array}{ccc}
0 & 0 & 0 \\
0 & 0 & -\omega J_{z} \\
0 & \omega J_{z} & 0
\end{array}\right]\left\{\begin{array}{c}
\dot{z} \\
\dot{\theta}_{x} \\
\dot{\theta}_{y}
\end{array}\right\}+} \\
& {\left[\begin{array}{ccc}
\sum_{i=1}^{6} k_{z_{i}} & -\sum_{i=1}^{6} k_{z_{i}} x_{i} \\
\sum_{i=1}^{6} k_{z_{i}} y_{i} & \sum_{i=1}^{6}\left(k_{y_{i}} z_{i}^{2}+k_{z_{i}} y_{i}^{2}+k_{m x_{i}}\right) & -\sum_{i=1}^{6} k_{z_{i}} x_{i} y_{i} \\
-\sum_{i=1}^{6} k_{z_{i}} x_{i} & -\sum_{i=1}^{6} k_{z_{i}} x_{i} y_{i} & \sum_{i=1}^{6}\left(k_{z_{i}} x_{i}^{2}+k_{x_{i}} z_{i}^{2}+k_{m y_{i}}\right)
\end{array}\right]=\left\{\begin{array}{c}
0 \\
m_{d} r_{d} h \omega^{2} \cos (\omega t) \\
m_{d} r_{d} h \omega^{2} \sin (\omega t)
\end{array}\right\}}
\end{aligned}
$$

Equation (10) was solved for various values of $k_{z_{i}}, i=1,2, \ldots, 6$. For the values of $k_{z_{i}}, i=$ $1,2, \ldots, 6$, shown in Table 2 , it was found that the Campbell diagram obtained from simulation (see figure 12) is very close to the Campbell diagram obtained from finite element analysis (see figure 11) 
and the experimentally obtained frequency values (see Table 1 ). The numerical values used in the simulations are: mass of the wheel, $m=8.1 \mathrm{~kg}$, inertias $J_{x}=J_{y}=0.06972 \mathrm{kgm}^{2}, J_{z}=0.12763$ $\mathrm{kgm}^{2}$, the static unbalance $m_{s} r_{s}=0.000072 \mathrm{~kg}-\mathrm{m}$, and the dynamic unbalance $m_{d} r_{d} h=3.01$ $\mathrm{kgm}^{2}$ [16]. The equation (10) and the parameters listed above constitute the lower-order model of the momentum wheel and the bracket. This model has been used in redesign studies which is discussed in section 4 .

It may be noted that the six spring stiffness values listed in Table 2 are different from the component mode synthesis values. This is expected as many spring stiffness terms were dropped in the simplified model. It may be further noted that even with this very simple model, with only six stiffness terms, a reasonably accurate prediction of the Campbell diagram was obtained.

Eigenvalue analysis of equation (10) gives three modes for different rotation speeds of wheel. This is plotted as Campbell diagram and the change of natural frequency with respect to the wheel speed is shown in figure 12. It can be seen that the mode of the wheel splits into negative whirl and positive whirl. The maximum response occurs when the wheel speed matches the whirl frequency. In the above condition it is seen that only one of the whirling modes are excited. This greatly amplifies the response and imparts large disturbance to the spacecraft, which in turn affects the scanning earth sensor output.

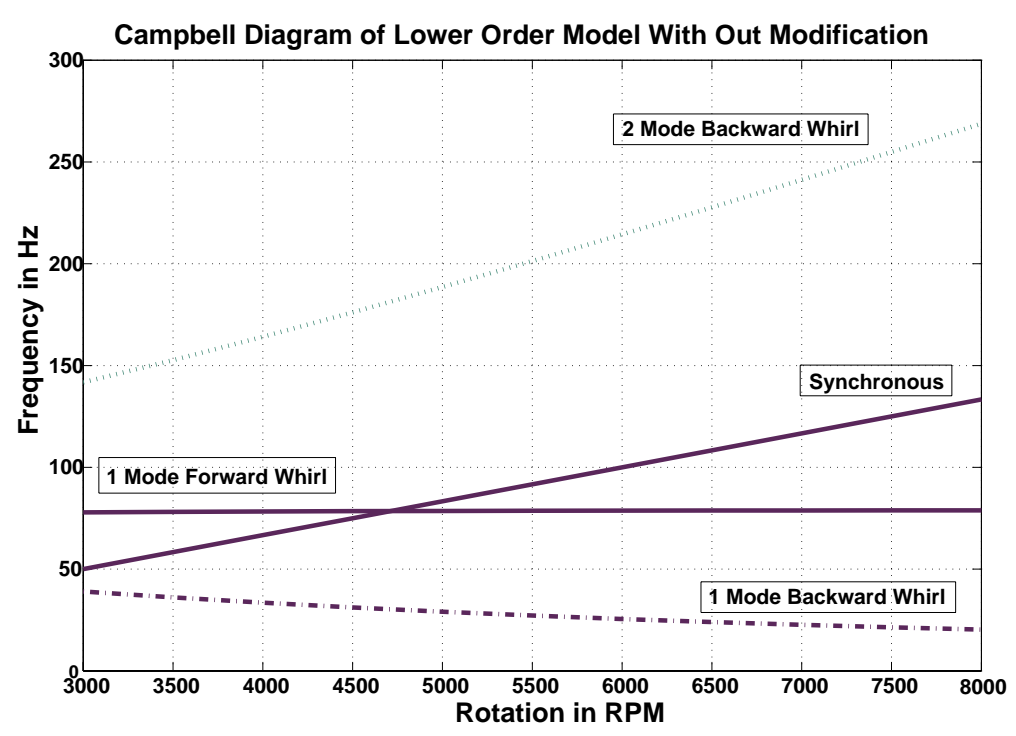

Figure 12: Campbell diagram from lower-order model 


\section{Redesign of the mounting bracket}

From equation (10), it is clear that only the $\theta_{x}$ and $\theta_{y}$ degrees of freedom influence the gyroscopic matrix. Hence to raise the whirling frequency beyond the operating range of speed of momentum wheel $(>5300 \mathrm{rpm})$, the stiffness corresponding to these degrees of freedom should be increased ${ }^{2}$. The stiffness that controls the $\theta_{x}$ and $\theta_{y}$ degrees of freedom are $k_{m x_{i}}, k_{m y_{i}}, i=1, \ldots, 6$. Keeping the stiffness values $k_{z_{i}}, i=1, \ldots, 6$ fixed, the values corresponding to $k_{m y_{i}}, i=1, \ldots, 6$, were varied to increase the whirl frequency from $4200 \mathrm{rpm}$ to $5600 \mathrm{rpm}$.

The final values of stiffness, $k_{z_{i}}, k_{m y_{i}}, i=1, \ldots, 6$, which satisfy the requirement are as follows:

$k_{z_{1}}=400000 \mathrm{~N} / \mathrm{m}, k_{z_{2}}=200000 \mathrm{~N} / \mathrm{m}, k_{z_{3}}=200000 \mathrm{~N} / \mathrm{m}$

$k_{z_{4}}=400000 \mathrm{~N} / \mathrm{m}, k_{z_{5}}=350000 \mathrm{~N} / \mathrm{m}, k_{z_{6}}=350000 \mathrm{~N} / \mathrm{m}$

$k_{m y_{1}}=10000 \mathrm{~N} / \mathrm{m}, k_{m y_{2}}=0 \mathrm{~N} / \mathrm{m}, k_{m y_{3}}=0 \mathrm{~N} / \mathrm{m}$

$k_{m y_{4}}=10000 \mathrm{~N} / \mathrm{m}, k_{m y_{5}}=10000 \mathrm{~N} / \mathrm{m}, k_{m y_{6}}=10000 \mathrm{~N} / \mathrm{m}$

The Campbell diagram corresponding to above values of stiffnesses is shown in figure 13 .

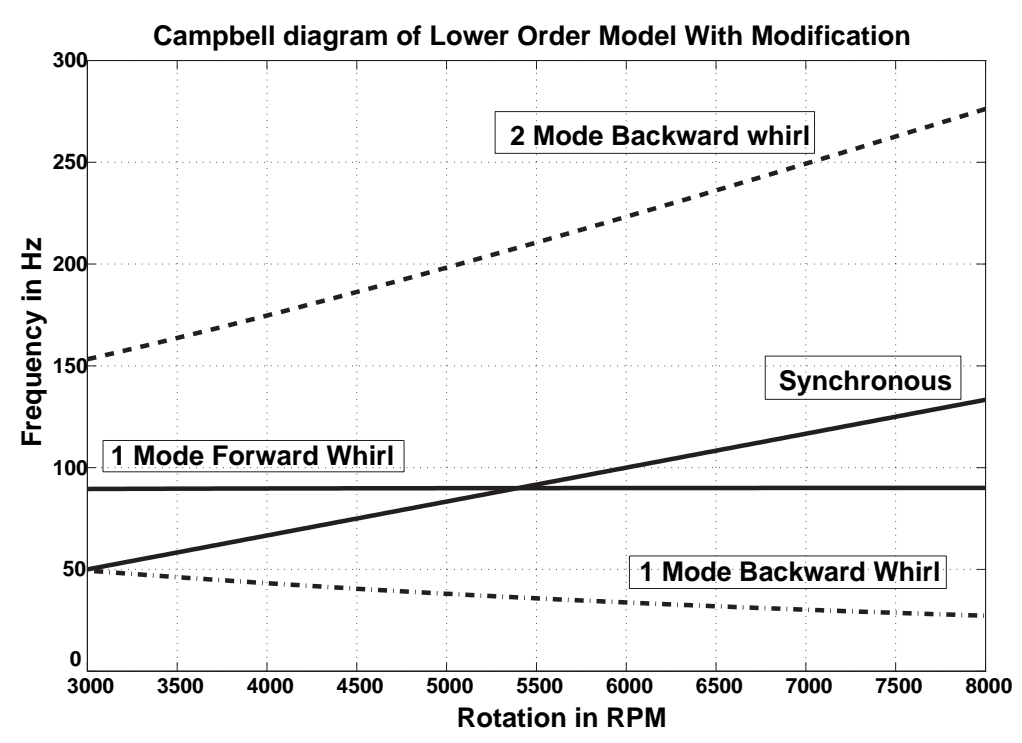

Figure 13: Campbell diagram from modified lower-order model

From the results of analysis of the lower-order model, it is clear that the gyroscopic forces plays important role in the dynamics of momentum wheel bracket especially when significant angular deformations are present at the mounting interface. It is also clear that the gyroscopic forces will affect the modes of the bracket causing whirling especially of those modes, which involve significant

\footnotetext{
${ }^{2}$ In this particular case, strengthening any other part of the bracket was difficult due to its design.
} 


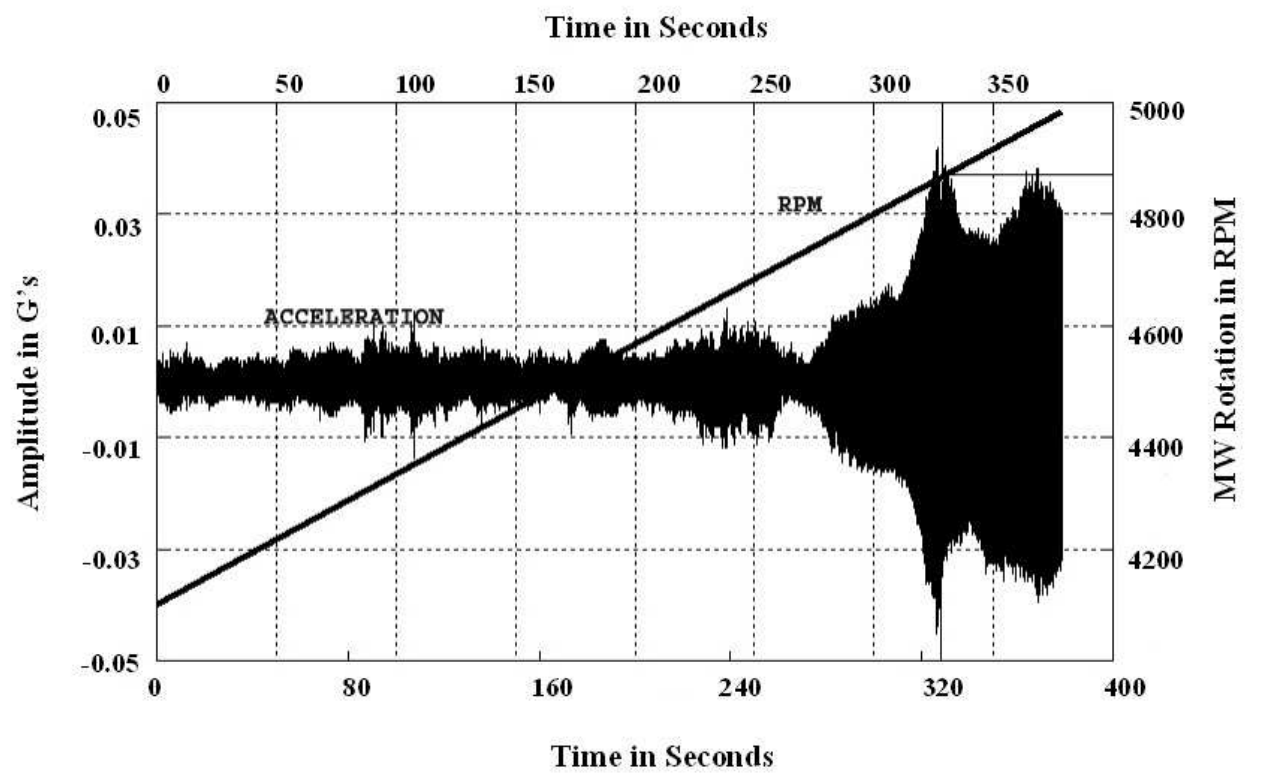

Figure 14: Response of the momentum wheel bracket after design modification

angular motion, viz., the anti-symmetric modes. Based on the results of analysis, it was decided to fix additional plates on either side of the milled bracket in a way so as to increase the stiffness $k_{m y_{i}}, i=1, \ldots, 6$. Although the analysis of the lower-order model and the values obtained for $k_{m y_{i}}, i=1, \ldots, 6$ do not have one to one correspondence with the geometry of the added plates, the lower-order model does indicate the role of stiffening by plates in increasing the critical whirl frequency.

After the modifications, the whirling frequency was shifted to around $82 \mathrm{~Hz}$ (4920 RPM) though the lower order model prediced it as $89 \mathrm{~Hz} 5300 \mathrm{RPM}$, as seen from the experimental data shown in figure 14. It was also observed that, this reduced the response near the earth sensor location to less than $0.1 \mathrm{~g}$ in the entire operating speed range of $3000 \mathrm{rpm}$ to $5100 \mathrm{rpm}$ compared to about 1.2 $\mathrm{g}$ before the modification.

\section{Conclusions}

This paper deals with the structural dynamic coupling of the rotating momentum wheel with the mounting bracket and its impact on the sensitive subsystems of a spacecraft. It is clearly brought out by experiments, finite element analysis and analysis using a lower-order model, that for the momentum wheel configuration studied, wheel rotation changes the natural frequency of the mounting bracket altering the frequency and amplitude of wheel disturbance itself. The influence 
of the rotational flexibility of the bracket is clearly brought out as the main factor influencing the coupling. A simplified low-order mathematical model of the wheel and bracket derived to study the influence of various stiffness terms is used for re-designing of the bracket. Finally, the changes on the bracket were finalised, and validated experimentally. The disturbing frequency was found to be greater than $89 \mathrm{~Hz}$, well beyond the operating range of momentum wheel. It must be stressed that although similar analysis could be have been done using a detailed finite element model, it would have been time consuming and expensive. The lower-order model can also be used for simulating the wheel bracket assembly in the integrated spacecraft.

\section{References}

[1] S. E. Woodard, The upper atmosphere research satellite in-flight dynamics, NASA-TM-110325, April 1997.

[2] S. E. Woodard, D. A. Gell, R. Lay, R. Jarnot, Experimental investigation of spacecraft inflight dynamic disturbances and dynamic response, Journal of Spacecraft and Rockets, 34(2), pp. 199-204, 1997.

[3] A. J. Butterfield, S. E. Woodard, Science instrument and structural interactions observed on the upper atmosphere research satellite, Journal of Spacecraft and Rockets, 33(4), pp. 556-562, 1996.

[4] S. E. Woodard, Orbital and configuration influences on spacecraft dynamic response, Journal of Spacecraft and Rockets, 35(2), pp. 177-182, 1998.

[5] D. F. Zimbelman, Thermal elastic shock, and its effect on Topex spacecraft attitude control, 14th Annual American Astronautical Society Guidance and Control Conference, Colorado, February 1991.

[6] T. Marshall, T. Gunderman and F. Mobley, Reaction wheel control of the MSX satellite, Proceedings of the Annual Rocky Mountain Guidance and Control Conference, AAS paper 91-038, pp. 119-138, 1991.

[7] R. A. Laskin and M. San Martin, Control/Structure system design of a space borne optical interferometer, Proceedings of the AAS/AIAA Astrodynamics Specialist Conference, AAS 89424, pp. 369-395, 1989.

[8] L. P. Davis, F. Wilson, R. E. Jewell, and J. J. Roden, Hubble space telescope reaction wheel assembly vibration isolation system, NASA Marshall Space Flight Center, March 1986. 
[9] S. Sabins, F. Schmitt and L. Smith, Magnetic reaction wheels, NASA Technical Report N7627336, 1976.

[10] T. Fukuda, H. Hosokai and N. Yajima, Flexibility control of solar battery arrays, Bulletin of the JSME 29, pp. 3121-3125, 1986.

[11] J. A. Bosgra and J. J. M Prins, Testing and investigation of reaction wheels, Automatic Control in Space (9th Symposium), pp. 449-458, 1982.

[12] An evaluation of reaction wheel emitted vibrations for large space telescope, NASA Technical Report N76-18213, 1976.

[13] B. Bialke, A compilation of reaction wheel induced spacecraft disturbances, $20^{\text {th }}$ Annual American Aeronautical Society Guidance and Control Conference, AAS paper 97-038, 1997.

[14] R. A. Masterson, Development, and validation of empirical and analytical reaction wheel disturbance models, Masters Thesis, Massachusetts Institute of Technology, June 1999.

[15] R. A Masterson, D. W. Miller and R. L. Grogan, Development and validation of reaction wheel disturbance models: Empirical model, Journal of Sound and Vibration, 249(3), pp. 575$598,2002$.

[16] INSAT-3B Spacecraft Handbook, ISRO-ISAC-INSAT-3B-PR-0150, February 2000.

[17] B. Bialke, High fidelity mathematical modelling of reaction wheel performance, Proceedings of the $21^{\text {st }}$ Annual AAS Rocky Mountance Guidance and Control Conference, AAS paper 98-0634, pp. 483-496, 1998.

[18] L. M. Elias, A structurally coupled disturbance analysis using dynamical mass measurement techniques, with application to spacecraft reaction wheel systems, Masters Thesis, Massachusetts Institute of Technology, March 2001.

[19] L. M. Elias, F. Dekens, I. Basdogan, L. Sievers, and T. Neville, A Methodology for modelling the mechanical interaction between a reaction wheel and a flexible structure, Proceedings of SPIE Astronomical Telescopes and Instrumentation Conference, Hawaii, August 2002.

[20] MSC NASTRAN (c) Reference Manual, V701, 2001.

[21] SDRC IDEAS (C) Test User's Manual, Master Series 2.1, 1996.

[22] R. R. Craig, and M. C. C. Bampton, Coupling of Substructures for Dynamic Analysis, AIAA Journal, (6)7, pp. 1313-1319, 1968. 
[23] M. J. Sidi, Spacecraft Dynamics and Control, Cambridge University Press, 1997. 